\title{
SIMULATION OF GAS-SOLID TWO-PHASE FLOW IN HORIZONTAL PIPE
}

\author{
Shigeru MATSUMOTO*, Shozaburo SAITO \\ AND SIRo MAEDA \\ Department of Chemical Engineering, Tohoku University, \\ Sendai, 980
}

\begin{abstract}
An ellipsoid bouncing model, previously presented to express the irregular bounce of particles on a duct wall, was extended to simulation of gas-solid suspension flow in a circular pipe. Simulated results were compared with experiments in terms of concentration distribution, particle velocity, additional pressure drop and frequency of particle collisions with the pipe wall. If the mean observed deformation, $\bar{\eta}_{o b}$, was taken as the value of the parameter, the mechanism of particle motion was explained for the most part by the simulation.
\end{abstract}

\section{Introduction}

Irregular bounce due to the non-spherical shape of particles and/or roughness of the duct wall has been proved to be the major mechanism for the motion of granular particles in horizontal gas-solid suspension flow $^{3-8)}$.

We have previously presented an ellipsoid bouncing model to express the irregular bounce of particles on the duct wall ${ }^{3}$. The ellipsoid bouncing model is based on the idea that a particle is approximated by an ellipsoid as shown in Fig. 1, and that the ellipsoid bounds two-dimensionally on the flat plate. The orientation of the particle at a position near the wall, that is angle $\varphi_{0}$ in Fig. 1, is assumed to be a probabilistic variable. On the basis of the model, the twodimensional motion of particles in a rectangular duct was satisfactorily simulated by the Monte Carlo method.

In this paper, an ellipsoid bouncing model which simulates the motion of particles in a circular pipe is described. Comparing simulated and experimental results, we concluded that the ellipsoid bouncing model can be applied to the simulation of suspension flow in a circular pipe.

\section{Procedures of Simulation}

\section{1 Equations of particle motion in a gas stream}

In terms of Cartesian coordinates $(x, y, z)$, as shown in Fig. 2, the component velocities of a particle are given by

$$
\boldsymbol{V}=\boldsymbol{i}_{x} V_{x}+\boldsymbol{i}_{y} V_{y}+\boldsymbol{i}_{z} V_{z}
$$

Received October 4, 1975.

Presented at the 9th General Symposium of The Soc. of Chem. Engrs., Japan, at Nagoya, Nov. 25, 1970. where $\boldsymbol{i}_{x}, \boldsymbol{i}_{y}$ and $\boldsymbol{i}_{z}$ are unit vectors in the direction of each of the three coordinates axes. From the same assumptions as in the previous paper ${ }^{3)}$, the equations describing particle motion in a gas stream are

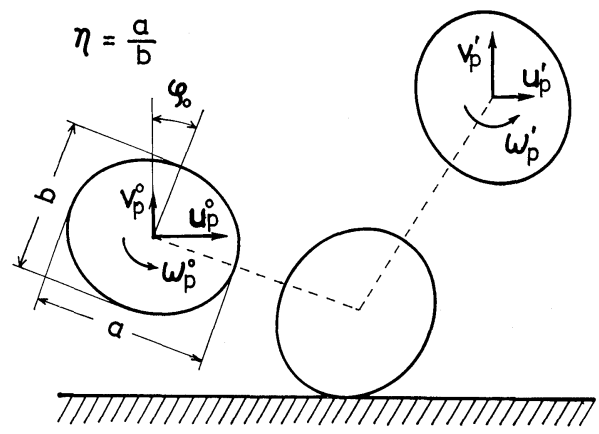

Fig. 1 Schematic representation of ellipsoid bouncing model

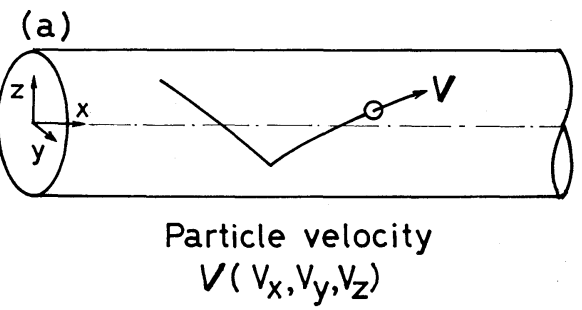

(b)

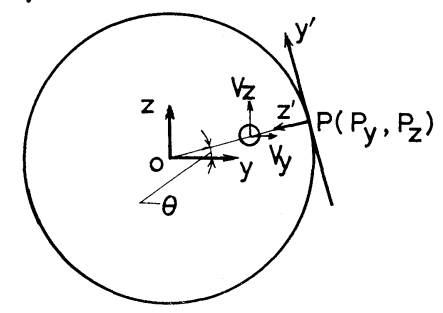

Fig. 2 Particle motion in horizontal pipe 


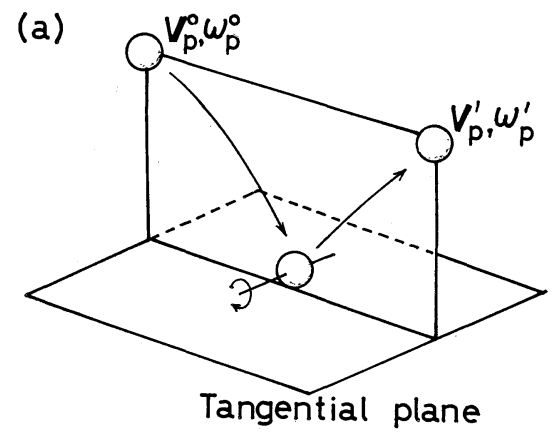

(b)

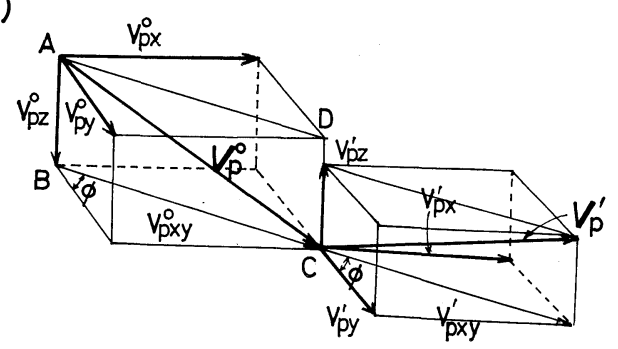

Fig. 3 Particle bouncing model in horizontal pipe

$$
\begin{aligned}
\frac{d V_{x}}{d t} & =\frac{3}{4} \frac{1}{d_{p}} \frac{\rho_{a}}{\rho_{p}} C_{D}{ }^{r_{r}}\left(u_{a}-V_{x}\right) \\
\frac{d V_{y}}{d t} & =-\frac{3}{4} \frac{1}{d_{p}} \frac{\rho_{a}}{\rho_{p}} C_{D^{\prime}{ }_{r}} V_{y} \\
\frac{d V_{z}}{d t}= & -\frac{3}{4} \frac{1}{d_{p}} \frac{\rho_{a}}{\rho_{p}} C_{D^{\prime}} r_{r} V_{z}+\left(\frac{\rho_{a}}{\rho_{p}}-1\right) g \\
& +\frac{3}{4} \frac{1}{d_{p}} \frac{\rho_{a}}{\rho_{p}} C_{L} \imath_{r}^{2}
\end{aligned}
$$

Air velocity $u_{a}$ in the above equations is given in the following power law form:

$$
\frac{u_{a}}{u_{a, \max }}=\left(1-\frac{r}{R}\right)^{1 / 7}
$$

where $r=\sqrt{y^{2}+z^{2}}$.

The drag coefficient $C_{D}$ and lift coefficient $C_{L}$ in Eqs. (2), (3) and (4) were calculated by the same procedures as in the previous paper.

\section{2 Bouncing of particle on the pipe wall}

Since the motion of the particle in a horizontal pipe is a three-dimensional motion, the axis of rotation does not always have a constant direction to the colliding plane. Hence, even a spherical particle does not show a simple bounce. In this investigation, this problem was simplified as follows: the axis of rotation was assumed to be always perpendicular to the trajectory plane of the particle and parallel to the bouncing plane, as shown in Fig. 3. The bouncing motion of the particle on the tangential plane of the pipe could then be calculated by the same procedures as those used in the case of a rectangular duct.

Let $V\left(V_{x}, V_{y}, V_{z}\right)$ denote the velocity of a particle approaching the pipe wall, and $V_{p}\left(V_{p x}, V_{p y}, V_{p z}\right)$ denote the particle velocity in the coordinate system $\left(x^{\prime}, y^{\prime}, z^{\prime}\right)$. The origin of the coordinates is taken at the point of contact, $P\left(P_{y}, P_{z}\right)$, and the $z^{\prime}$-axis is perpendicular to the tangential plane, as shown in Fig. 2. Then, $\boldsymbol{V}$ is transformed to $\boldsymbol{V}_{p}$ as follows:

$$
\begin{aligned}
& V_{p x}=V_{x} \\
& V_{p y}=-\sin \theta \cdot V_{y}+\cos \theta \cdot V_{z} \\
& V_{p_{z}}=-\cos \theta \cdot V_{y}-\sin \theta \cdot V_{z}
\end{aligned}
$$

In the above equations, the azimuthal angle $\theta$ is defined as shown in Fig. 2 (b) and is related to the position of the particle with coordinates $(x, y, z)$ as follows:

$$
\theta=\arctan (z / y)
$$

Using Eqs. (6), (7) and (8), the velocity vector of the particle approaching the bouncing plane, $V_{p}^{\circ}\left(V_{p x}^{\circ}, V_{p y}^{\circ}\right.$, $V_{p z}^{\circ}$ ), can be obtained.

Now considering a two-dimensional bouncing motion $\left(V_{p x y}^{\circ}, V_{p z}^{\circ}\right)$ for the particle in the trajectory plane, ABCD in Fig. 3 (b), the velocity component $V_{p x y}^{\circ}$ is given by

$$
V_{p x y}^{\circ}=\sqrt{V_{p x}^{\circ 2}+V_{p y}^{\circ 2}}
$$

These velocity components $\left(V_{p x y}^{\circ}, V_{p z}^{\circ}\right)$, and angular velocity $\omega_{p}^{\circ}$, correspond to the velocity components $u_{p}^{\circ}, \imath_{p}^{\circ}$ and $\omega_{p}^{\circ}$ in Fig. 1. Therefore, velocity components $\left(V_{p x y}^{\prime}, V_{p z}^{\prime}\right)$ and angular velocity $\omega_{p}^{\prime}$ after bounce can be calculated by the same procedure as in the previous paper. Equations of impulsive motion and their solution are abbreviated since they were previously presented.

If the angle between the two velocity components, $V_{p x}^{\circ}$ and $V_{p y}^{\circ}$, is designated by $\phi$ in Fig. $3(\mathrm{~b})$, the velocity components, $V_{p x}^{\prime}$ and $V_{p y}^{\prime}$, after bounce can be obtained by

$$
V_{p x}^{\prime}=V_{p x y}^{\prime} \cdot \sin \phi
$$

and

$$
V_{p y}^{\prime}=V_{p x y}^{\prime} \cdot \cos \phi
$$

where $\phi=\arctan \left(V_{p x}^{\circ} / V_{p y}^{\circ}\right)$.

Finally, the velocity components after bounce, $\left(V_{p x}^{\prime}, V_{p y}^{\prime}, V_{p z}^{\prime}\right)$, in the tangential plane coordinate system are tansformed conversely to those in the fixed coordinate system by using Eqs. (6), (7) and (8).

\section{3 Initial conditions}

For the initial conditions of simulation, particles at the feed section are assumed to be distributed uniformly at the top of that pipe and to fall into the pipe by gravity alone. The initial condition of particle motion can then be given as follows: first a set of two pseudo-random variables, $\left(X_{r}, Y_{r}\right)$, in the range from $-R$ to $R$, which satisfy the condition $X_{r}^{2}+Y_{r}^{2}<R^{2}$ were generated. Let $x_{0}=X_{r}$, and $y_{0}=Y_{r}$. Then, $z_{0}$ is calculated by

$$
z_{0}=\sqrt{R^{2}-y_{0}^{2}}
$$

The falling velocity, $V_{z, 0}$, in this position is

$$
V_{z, 0}=-\sqrt{2 g z_{0}}
$$


Consequently, the initial velocity of the particle is $\left(0,0, V_{z, 0}\right)$.

\section{4 Procedure of simulation}

The concentration distribution of particles was computed from the residence time distribution of particles as in the previous paper. An additional pressure drop of two-phase flow was obtained on the basis of the assumption that the additional pressure drop was mainly due to the loss of kinetic energy of particles during collision with the pipe wall. The statistics of frequencies of particle collision with the pipe wall in the simulation were also recorded.

\section{Experimental Apparatus and Procedures}

The experimental apparatus was almost the same as that previously used ${ }^{11}$. In brief, it was a pressuretype conveying line consisting of an $11 \mathrm{~m}$ length of $26 \mathrm{~mm}$ I.D. Pyrex-glass pipe. The concentration and velocity of particle were measured photographically. Frequencies of particle collisions with the pipe wall were measured by means of a semiconductor pressure transducer. The semiconductor pressure transducer possesses fine sensitivity and excellent frequency characteristics, with an upper limit of about $40 \mathrm{kHz}$. Hence it can be used to measure the frequency of particle collisions at a position on the pipe wall. The mounting of the probe is illustrated in Fig. 4. The arrangement permits the probe to be rotated without shutting down the system. It was confirmed with visual inspection that this instrument scarcely had any influence on the motion of particles in the stream. Output signals from the pressure transducer were detected by a synchroscope and recorded by photographing the images appearing on the synchroscope. We took statistics of about 500 pulses which were obtained from about 40 photographic sheets and calculated the average frequency of particle collisions per unit time. From the preparatory experiment, it was found that pulses were not detected by the synchroscope when a particle collided with the pipe wall apart from the probe itself, and were detected only when it collided with the probe.

The physical properties of particle used in this experiment are shown in Table 1.

\section{Calculated Results}

\section{1 Particle trajectory in horizontal pipe}

Before applying the ellipsoid model, the motion of a particle was simulated by the spherical particle model, which was the same as the ellipsoid model except that the shape of particle was spherical. From this simulation, it can be shown how far a particle can be transported by lift force alone. However, since the trajectory of a particle depends on the initial conditions, there is no general expression to show the

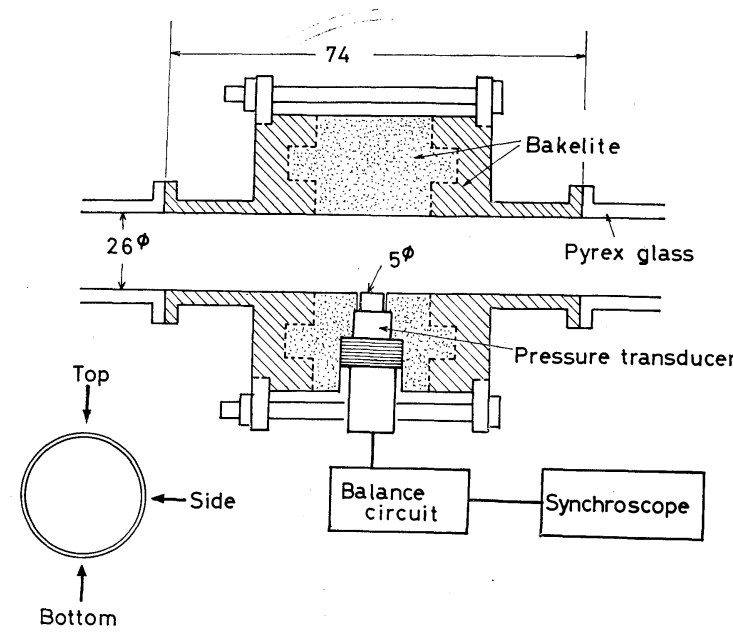

Fig. 4 Experimental apparatus for measuring frequency of particle collisions with pipe wall

\begin{tabular}{|c|c|c|c|c|c|}
\hline \multirow[b]{2}{*}{ Materials } & \multirow{2}{*}{$\begin{array}{c}\text { Table } 1 \\
\rho_{p} \\
{\left[\mathrm{~g} / \mathrm{cm}^{3}\right]}\end{array}$} & \multicolumn{3}{|c|}{ Properties of particles } & \multirow[b]{2}{*}[\overline{\eta}_{ob}]{} \\
\hline & & $\begin{array}{c}d_{p} \\
{[\mathrm{~mm}]}\end{array}$ & $\stackrel{e}{-]}]$ & $\stackrel{\mu_{k}}{[-]}$ & \\
\hline Glass & 2.5 & 1.00 & 0.97 & 0.4 & 1.02 \\
\hline Glass & 2.5 & 0.50 & 0.97 & 0.4 & 1.02 \\
\hline Glass & 2.5 & 0.17 & 0.97 & 0.4 & 1.02 \\
\hline Copper & 8.9 & 0.27 & 0.85 & 0.53 & - \\
\hline
\end{tabular}

particle trajectory. Hence, we draw the trajectory projected on the sectional plane of the pipe at a certain distance. Fig. 5 shows an example of trajectories computed for $1.0 \mathrm{~mm}$ glass beads at $\bar{u}_{a}=7 \mathrm{~m} / \mathrm{sec}$. Whatever position of the inlet section a particle is put into, it is found that the particle settles out on the bottom at about 2 meters from the feed point. For example, a $0.1 \mathrm{~mm}$ glass bead at an air velocity of $10 \mathrm{~m} / \mathrm{sec}$ could not float farther than $3 \mathrm{~m}$. Thus, it was found that particles could not keep floating by lift force alone in the case of a circular pipe. Fig. 6 shows an example of particle trajectories computed on the ellipsoid bouncing model. The condition for simulation is the same as in Fig. 5, that is, $1.0 \mathrm{~mm}$ glass bead and $\tilde{u}_{a}=7 \mathrm{~m} / \mathrm{sec}$. The parameter for the ellipsoid bouncing model, $\eta$, which is the ratio of major and minor axes of the ellipsoid, is 1.05. In this case, it can be seen that the particle collides frequently with the pipe wall and keeps floating owing to an irregular bounce.

\section{2 Distributions of concentration and velocity of} particles, and additional pressure drop

First, a number of trials using the Monte Carlo simulation, $N$, were investigated as shown in Fig. 7. Although 50 trials were enough for the case of the rectangular duct, it was found that in this case at least about 70 trials were necessary because of the randomness included in the initial conditions. Finally, in this simulation, 100 trials were carried out. Further, statistics of concentration distribution and other 


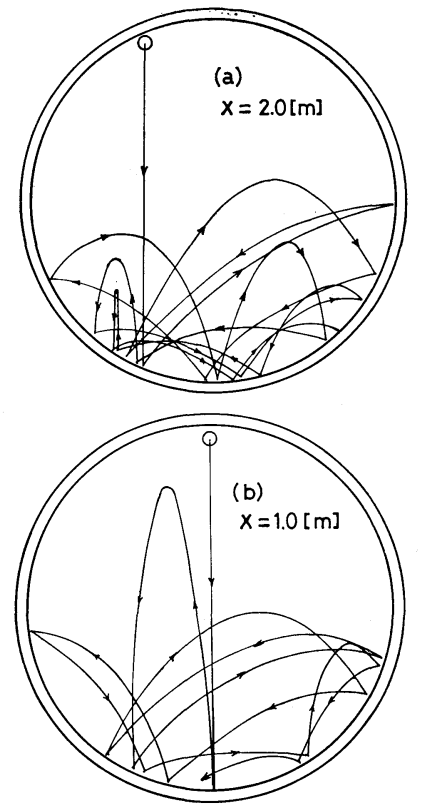

Fig. 5 Trajectories of a spherical particle in horizontal pipe $\left(D=2.6[\mathrm{~cm}]\right.$, glass bead, $d_{p}=1.0[\mathrm{~mm}], \bar{u}_{a}=7[\mathrm{~m} /$ sec])

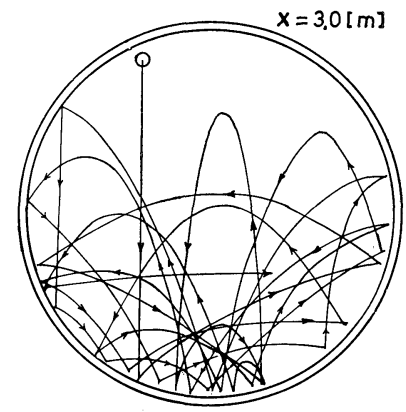

Fig. 6 Trajectory of an ellipsoid particle in horizontal pipe $\left(D=2.6[\mathrm{~cm}]\right.$, glass bead, $d_{p}=1.0[\mathrm{~mm}], \quad \eta=1.05$, $\left.\overline{\boldsymbol{u}}_{a}=7[\mathrm{~m} / \mathrm{sec}]\right)$

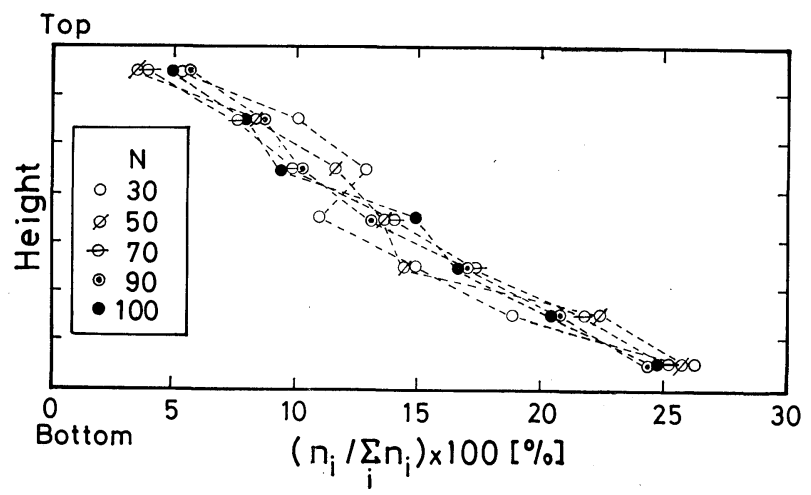

Fig. 7 Effect of $N$ on the concentration distribution of particles at $x=5 \mathrm{~m} \quad\left(D=2.6[\mathrm{~cm}], d_{p}=1.0[\mathrm{~mm}]\right.$, glass beads, $\left.\boldsymbol{\eta}=1.02, \bar{u}_{a}=7[\mathrm{~m} / \mathrm{sec}]\right)$

quantities were taken at every ten trials, to confirm convergency. Fig. 8 shows how the concentration distribution of particles varies with distance from the feed point up to $x=5 \mathrm{~m}$. In this figure, the distribution of particles becomes nearly constant at $x=$

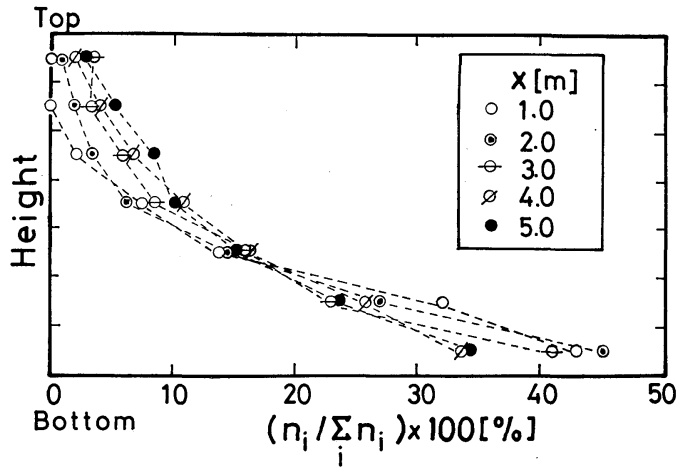

Fig. 8 Effect of $x$ on the concentration distribution of particles in horizontal pipe $\left(D=2.6[\mathrm{~cm}]\right.$, glass beads, $d_{p}=$ $\left.1.0[\mathrm{~mm}], \eta=1.02, \bar{u}_{a}=7[\mathrm{~m} / \mathrm{sec}]\right)$

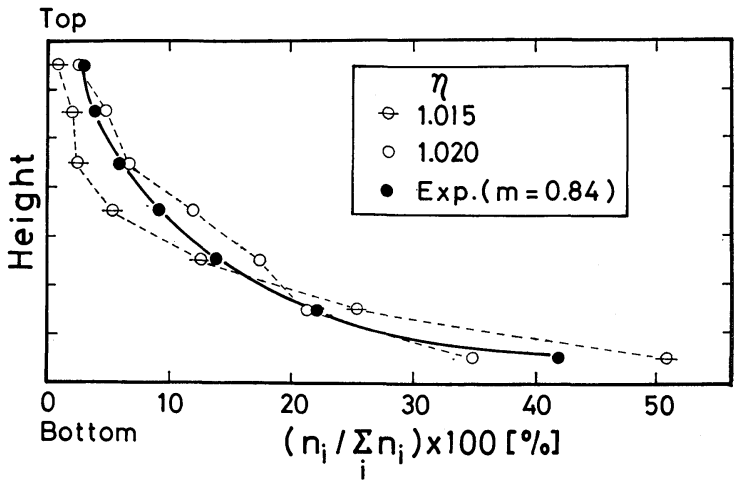

Fig. 9 Effect of $\eta$ on the concentration distribution of particles $\left(D=2.6[\mathrm{~cm}]\right.$, glass beads, $d_{p}=1.0[\mathrm{~mm}], \vec{u}_{a}=7$ $[\mathrm{m} / \mathrm{sec}])$

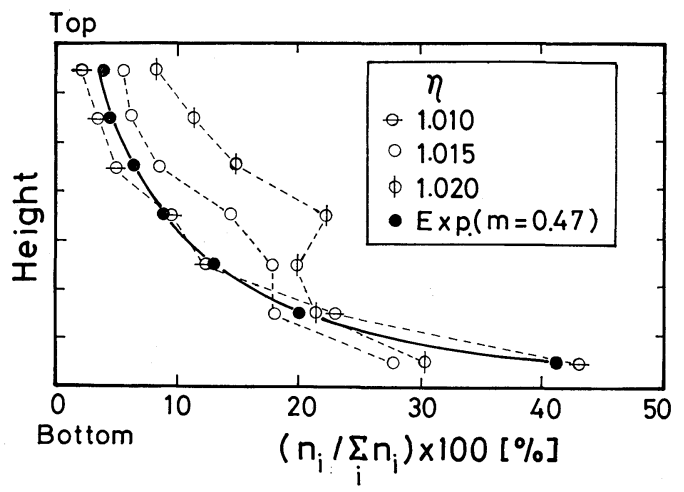

Fig. 10 Effect of $\eta$ on the concentration distribution of particles $\left(D=2.6[\mathrm{~cm}]\right.$, glass beads, $d_{p}=0.5[\mathrm{~mm}], \bar{u}_{a}=10$ $[\mathrm{m} / \mathrm{sec}])$

$4 \mathrm{~m}$. The effects of $\eta$ on the concentration distribution are illustrated in Figs. $\mathbf{9}$ and $\mathbf{1 0}$ in comparison with experimental results. Figs. 11 and $\mathbf{1 2}$ show, respectively, the effects of $\eta$ on the translational velocity of particle and the additional pressure drop. In these figures, the calculated results are in fair agreement with experiments when $\eta=1.015$ or 1.020 , which is nearly equal to the mean value of observed deformation, $\bar{\eta}_{o b}$. In the case of particle velocity, the difference between calculated and experimental results seems to be wide, but the calculated result 


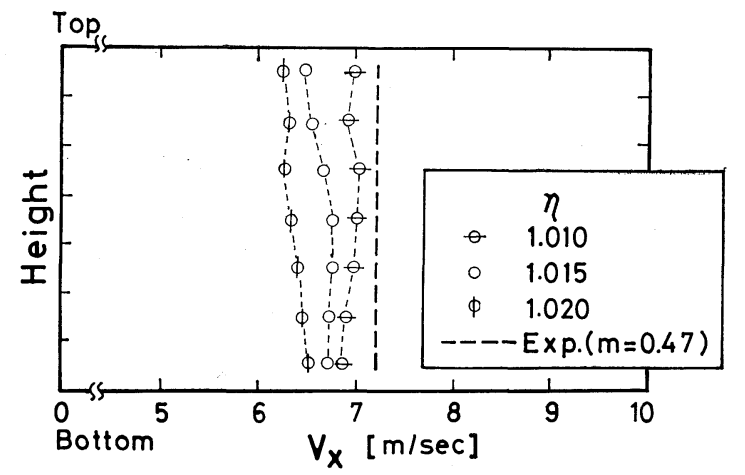

Fig. 11 Effect of $\eta$ on the particle velocity (The set of parameters is the same as Fig. 10)

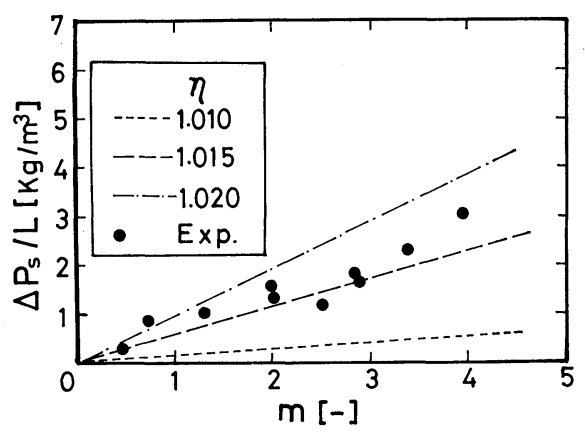

Fig. 12 Effect of $\eta$ on the additional pressure drop (The set of parameters is the same as Fig. 10)

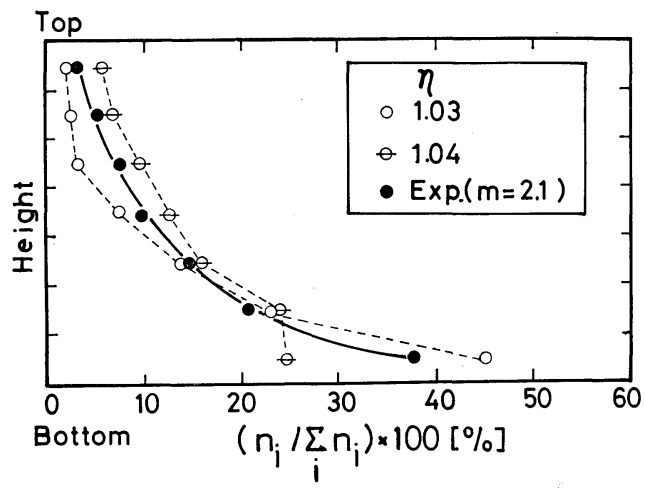

Fig. 13 Effect of $\eta$ on the concentration distribution of particles $\left(D=4.68[\mathrm{~cm}]\right.$, copper beads, $d_{p}=0.3[\mathrm{~mm}], \overline{\boldsymbol{u}}_{a}=$ $10[\mathrm{~m} / \mathrm{sec}])$

when $\eta=1.015$ lies within the range of experimental error since the measurement of particle velocity contains an error of about $\pm 7 \%$. Fig. 13 shows the calculated results for $0.3 \mathrm{~mm}$ copper beads when $\bar{u}_{a}=10 \mathrm{~m} / \mathrm{sec}$ and $D=46.8 \mathrm{~mm}$. The observed value in this figure was quoted from a thesis by Konno ${ }^{2)}$. Although $\bar{\eta}_{o b}$ for these beads is not known, the value of the parameter $\eta$ in which the calculated value agrees well with the experimental one is reasonable.

3. 3 Frequency of particle collisions with the pipe wall

Frequencies of particle collisions with the pipe wall were calculated in the simulation and compared with

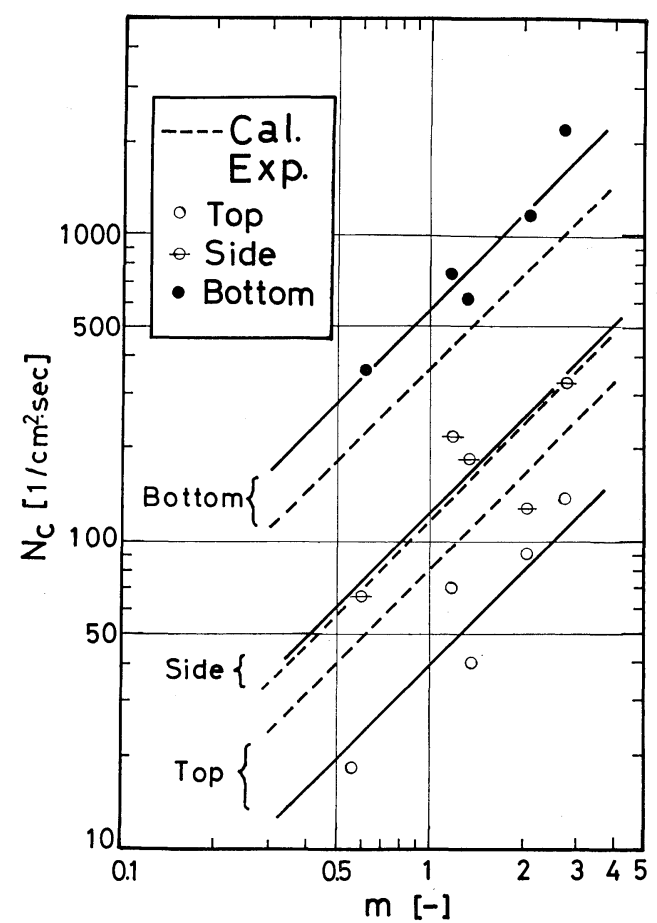

Fig. 14 Frequencies of particle collisions with pipe wall $\left(D=2.6[\mathrm{~cm}]\right.$, glass beads, $d_{p}=0.5[\mathrm{~mm}], \bar{u}_{a}=8[\mathrm{~m} / \mathrm{sec}], \eta=$ 1.02)

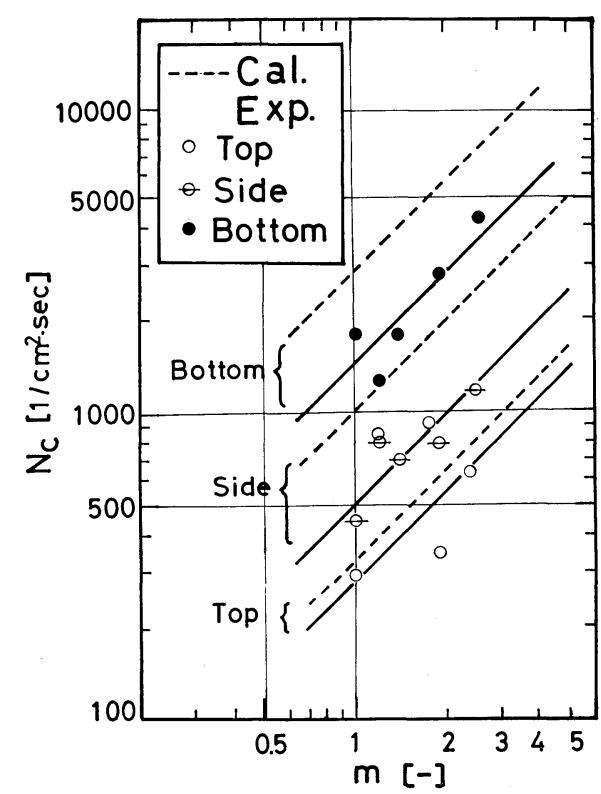

Fig. 15 Frequencies of particle collisions with pipe wall $\left(D=2.6[\mathrm{~cm}]\right.$, glass beads, $d_{p}=0.2[\mathrm{~mm}], \eta=1.02, \bar{u}_{a}=8$ $[\mathrm{m} / \mathrm{sec}])$

experimental results, to get more detailed information on particle motion in the pipe. Fig. 14 shows the influence of mass flow ratio, $m$, on the frequency of particle collision per unit area, $N_{c}$, when transporting $0.5 \mathrm{~mm}$ glass beads at $\bar{u}_{a}=8 \mathrm{~m} / \mathrm{sec}$. It is obvious from this figure that the observed frequency becomes greater in the order top, side, and bottom of pipe. As regards the top and bottom, the calculated results 
are slightly different from the experiments, but calculations for the side show good agreement with observed values. Within the range of these experiments, the observed value for $N_{c}$ is nearly proportional to mass flow ratio. This signifies that $N_{c}$ is almost proportional to the flux of particle number. Fig. 15 shows the comparison of frequency of particle collisions when transporting $0.2 \mathrm{~mm}$ glass beads at $\bar{u}_{a}=8 \mathrm{~m} / \mathrm{sec}$. In this case, the calculated values for the bottom and side locations are higher than the observed values, but they are of the same order of magnitude. These discrepancies are considered to be caused by the fact that particle-diameter distribution was not taken into account in the simulation, although frequency of collision might be affected greatly by particle diameter. Further, the number of Monte Carlo trials, which was 100 in this simulation, is perhaps insufficient for the statistics of collision frequency.

Generally, the observed values for frequency of particle collisions almost agreed with Mori and Suganuma's experimental equation ${ }^{5)}$.

\section{Conclusion}

The ellipsoid bouncing model previously presented was extended to the simulation of gas-solid suspension flow in a circular pipe. When the mean observed deformation, $\bar{\eta}_{o b}$, was taken as the value of the parameter, the concentration distribution of particles and additional pressure drop were simulated satisfactorily. However, the particle velocity and frequency of particle collision were partially different from the experiments because of incompleteness in the simulation model.

\section{Nomenclature}

$\begin{array}{lll}a, b & = & \text { length of the major and minor axes of a } \\ & \text { ellipsoid } \\ = & \text { drag coefficient } \\ C_{D} & = & \text { lift coefficient } \\ C_{L} & = & \text { pipe diameter } \\ D & = & \text { particle diameter } \\ d_{p} & = & \text { coefficient of restitution } \\ e & = & \text { gravitational acceleration } \\ g & = & \text { Cartesian unit vectors } \\ \boldsymbol{i}_{x}, \boldsymbol{i}_{y}, \boldsymbol{i}_{z} & = & \text { pipe length } \\ L & = & \text { mass flow ratio of solid to fluid } \\ m & = & \text { number of Monte Carlo trials } \\ N & & \text { pipe wall of particle collisions with } \\ N_{C} & \end{array}$

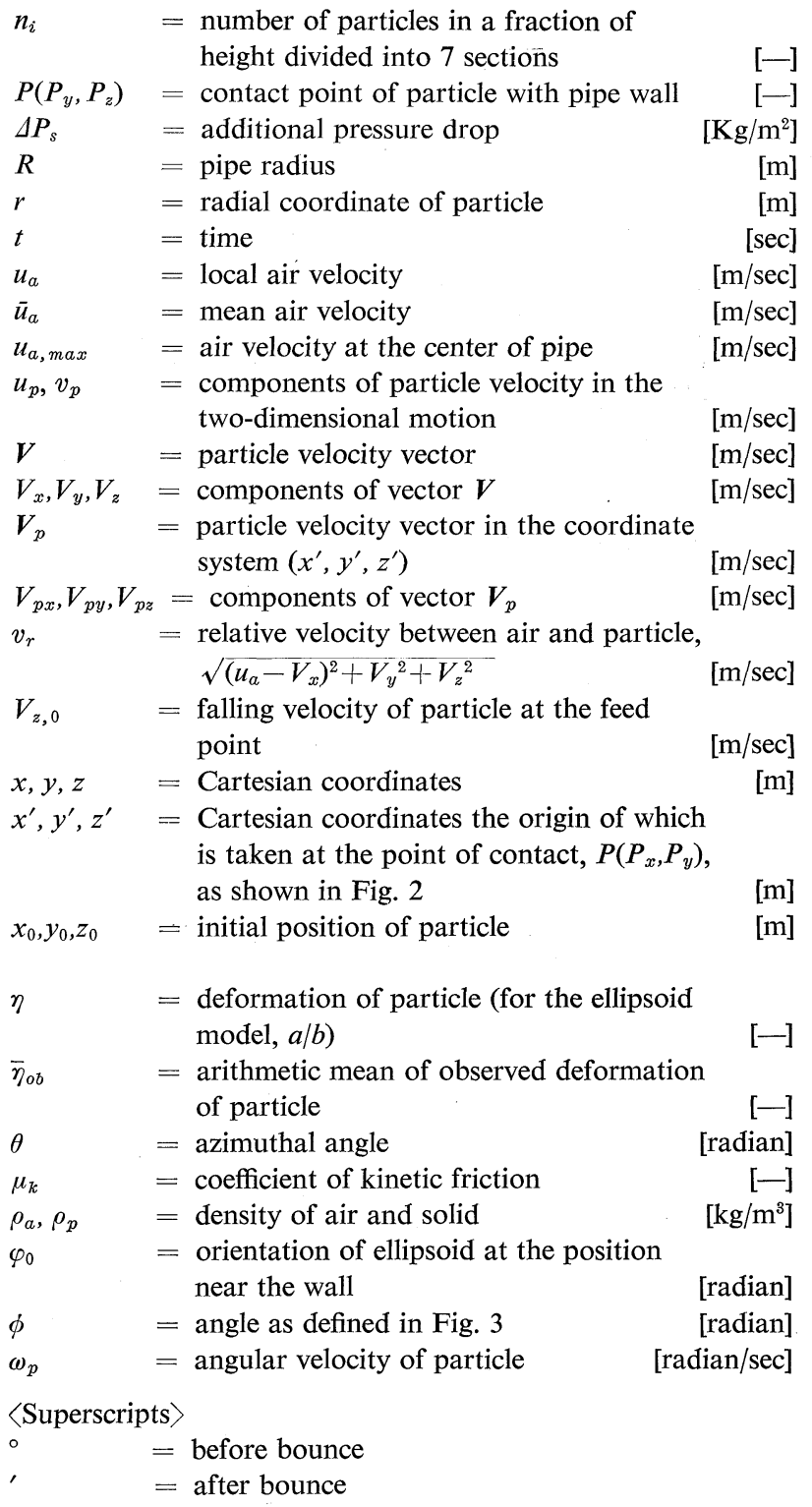

\section{Literature Cited}

1) Konno, H., S. Saito and S. Maeda: Kagaku Kögaku, 31, 243 (1967).

2) Konno, H.: Ph. D. thesis, Tohoku University (1969).

3) Matsumoto, S. and S. Saito: J. Chem. Eng. Japan, 3, 83 (1970).

4) Matsumoto, S. and S. Saito: ibid., 3, 223 (1970).

5) Mori, Y. and A. Suganuma: Kagaku Kögaku, 29, 615 (1965).

6) Mori, Y., A. Suganuma and H. Sadotomo: Preprint of 31st Annual Meeting of The Soc. of Chem. Engrs., Japan (1966).

7) Muschelknautz, E.: V.D.I. Forschungsheft, 476 (1959).

8) Ranz, W. E., G. R. Talandis and B. Gutterman: AIChE J., 6, 124 (1960). 